\title{
Role of MRI Evaluation in Knee Injuries
}

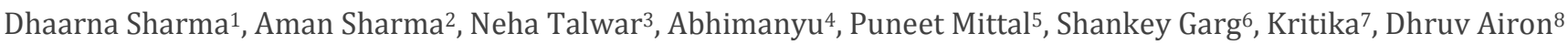

${ }^{1}$ Department of Radiology, MMIMSR, Mullana, Haryana, India. ${ }^{2}$ Department of Radiology, MMIMSR, Mullana, Haryana, India. ${ }^{3}$ Department of Medicine, MMIMSR, Mullana, Haryana, India. ${ }^{4}$ Department of Medicine, MMIMSR, Mullana, Haryana, India. ${ }^{5}$ Department of Radiology, MMIMSR, Mullana, Haryana, India. ${ }^{6}$ Department of Radiology, MMIMSR, Mullana, Haryana, India. ${ }^{7}$ Department of Radiology, MMIMSR, Mullana, Haryana, India. ${ }^{8}$ Department of Radiology, MMIMSR, Mullana, Haryana, India.

\section{ABSTRACT}

\section{BACKGROUND}

Knee is a major weight bearing and largest joint that provides mobility and stability during physical activities as well as balance while standing. Due to its wide range of functions it is exposed to forces beyond its physiological range, and thus bone and soft tissue of knee are at risk of injuries. We wanted to study MRI appearances of cruciate ligaments and meniscal tears in cases of knee injuries and correlate the MRI findings of knee injury with clinical observations/other radiological investigations/ arthroscopic findings. We also wanted to evaluate the sensitivity, specificity, PPV, NPV and accuracy of MRI in detecting knee injuries taking arthroscopy as gold standard (wherever performed).

\section{METHODS}

This prospective diagnostic descriptive study was conducted in the Department of Radiodiagnosis of Maharishi Markandeshwar Institute of Medical Sciences and Research, MMDU, Mullana, among 100 patients presenting with knee injuries referred to Department of Radiodiagnosis for MR imaging. MR scans were carried out on 1.5 tesla MR machine Achieva (by Phillips Medical System) and studies were performed with the sense extremity coil. Arthroscopic knee surgery was performed in 39 patients. MR findings were correlated with clinical and arthroscopic findings wherever possible.

\section{RESULTS}

MR diagnosis of 100 cases was as follows: in ACL tear 50\%; PCL tear 19\%; MCL tear 17\%; LCL tear 16\%; MM tear 49\%; LM tear 31\%; Sensitivity, specificity, NPV, PPV and accuracy of MRI in detecting the knee injuries taking arthroscopy as gold standard are as follows ACL- 100\%, 90.9\%, 100\%, 96.5\% and $97.4 \%$; lateral meniscus-100\%, 94.5\%, 100\%, 50\% and 94.8\%; medial meniscus $90.4 \%, 66.7 \%$, $85.7 \%, 76 \%$ and $79.5 \%$ respectively. Correlation between MRI and arthroscopic findings regarding presence and absence of posterior cruciate ligament tear was highest with sensitivity $-100 \%$, specificity- $94.5 \%$, NPV- $100 \%$ and accuracy-94.8\%, There were 6 false positives for meniscus tear on MR examinations accounting for low positive predictive value (72\%) of MR examination. Posterior horn tears of menisci are likely to be missed on arthroscopy. Out of 6 false positive cases of meniscus tear, 4 were located predominately in the posterior horn and 2 in the anterior horn. Thus, acceptance of MRI findings as false positive is controversial.

\section{CONCLUSIONS}

MRI is a useful non-invasive modality having high diagnostic accuracy, sensitivity and negative predictive value making it a very reliable screening test for diagnosis of internal derangements of knee joint. Thus, MRI saves many knees from unnecessary arthroscopies.

\section{KEY WORDS}

Arthroscopy, Knee Injury, MRI
Corresponding Author: Dr. Dhaarna Sharma, $1^{\text {st }}$ Year Postgraduate Resident, Department of Radiology, MMIMSR, Mullana-133207, Haryana, India.

E-mail: dhaarnasharma60@gmail.com

DOI: $10.14260 /$ jemds/2020/313

Financial or Other Competing Interests: None.

How to Cite This Article:

Sharma D, Sharma A, Talwar N, et al. Role of MRI evaluation in knee injuries. J. Evolution Med. Dent. Sci. 2020;9(17): 1435-1441, DOI:

10.14260/jemds/2020/313

Submission 18-02-2020,

Peer Review 15-04-2020,

Acceptance 22-04-2020,

Published 27-04-2020.

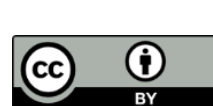




\section{BACKGROUND}

Knee is a major weight bearing and largest joint that provides mobility and stability during physical activity as well as balance while standing. It is also the main joint for sports that involve running, jumping, kicking and changing directions. Due to its wide range of function, the joint relies on multiple soft tissue structures to maintain bony alignment during weight bearing and movement. If the knee is exposed to forces beyond its physiological range, bone or soft tissue are at risk of injury. (1) Knee injury represents roughly $6 \%$ of all acute injuries treated at emergency department. Knee pain is a complaint in $20 \%$ of the general adult population and trauma to the knee is 2nd most common occupational accident. $15 \%$ of all sports injuries involve the knee. $75 \%$ of the surgeries done on professional football players involve ligament tear and meniscal injuries. The knee injury risk is especially high in the age group of 15 to 25 years, especially in various team sports and ball games. $(2,3)$

Accurate and timely diagnosis increases the likelihood of fully restoring normal and pain free use of affected knee. Physical examination of painful knee in acute phase may be difficult and frequently imaging studies are required to aid in the assessment of these injuries. Plain radiography is the most readily available and least expensive imaging modality, but it lacks sensitivity for detection of meniscus, cartilage, bone marrow and ligamentous injuries. Use of arthrography and arthroscopy improves the accuracy of diagnosis but both are invasive and cause complications and moreover these techniques require a skilled person for reporting and interpreting the results. $(4,5)$

Magnetic Resonance Imaging [MRI], a non-invasive modality, is now routinely used to assess a wide spectrum of internal knee derangement. Potential of MRI in assessing the knee injuries were first reported in 1983 in literature. Since then it has changed the traditional algorithm for work up of knee joint pathology, particularly when meniscal and cruciate ligament injuries are suspected. Additional advantages of MRI are multiplanar and thin section capabilities. It does not utilise ionizing radiation therefore it is entirely safe. It is also non-invasive, painless and allows acquisition of images in multiple planes without repositioning the patient. In addition, MRI provides excellent spatial and contrast resolution of both intra and extra articular structure. Thus, MRI clearly emerged as primary imaging tool in work up of knee joint pathology. (6)

We wanted to study MRI appearances of cruciate ligaments and meniscal tears in cases of knee injury, correlate the MRI findings of knee injury with clinical observations/other radiological investigations/ arthroscopic findings and determine the sensitivity, specificity, PPV, NPV and accuracy of MRI in detecting knee injuries taking arthroscopy as gold standard (wherever performed).

\section{METHODS}

This study was a prospective, diagnostic descriptive study conducted in the department of Radio diagnosis of Maharishi Markandeshwar, Institute of Medical Sciences and Research,
Mullana. This study was conducted between January 2013 to June 2014. 100 patients presenting with knee injury referred from OPD (outpatient Department) \& IPD (inpatient Department) of Orthopaedics to the Department of Radio diagnosis for MR imaging were included in the study, Arthroscopy was done in 39 patients and findings of arthroscopy were correlated with MRI findings. Sensitivity, specificity NPV, PPV and accuracy of MRI were calculated.

\section{Inclusion Criteria}

All patients of knee injury to either one or both knees were undergone MR Imaging.

\section{Exclusion Criteria}

1. Patient with contraindications to MRI.

2. Patient with prior arthroscopy or surgical intervention.

3. Patient with known joint disease e.g.: Neoplasm, inflammatory or infectious disorder.

4. Patient with history of old significant trauma to the currently injured Knee

Once the patient satisfies the criteria for this study, a written informed consent was obtained from each patient and a detailed history was elicited from all the patients. Relevant Past history was also recorded. Findings of general physical and specific systemic examination were recorded in detail. X-ray knee joint (AP and lateral view) were done and findings were recorded. Any other view of knee joint e.g. oblique view etc., if done was documented. Then the MRI was done, and findings were also correlated with clinical diagnosis. MR findings were recorded in a Performa and correlated with clinical observations/ other radiological investigations/arthroscopic findings (wherever preformed)

\section{MRI Protocol}

MR scans were carried out on 1.5 tesla MR machine Achieva (by Phillips Medical System) with sense extremity coil (Philips Medical System). Patient was placed in supine position and feet first in MR imager, with knee to be imaged in approximately 15-20-degree external rotation to aid the imaging of anterior cruciate ligament in the sagittal plane. Knee was also flexed 5-10 degrees for assessing patellofemoral compartment... Knee to be imaged was centred with the $160 \mathrm{~mm}$ field of view, including in the image both the suprapatellar bursa and insertion of patellar ligament on the tibial tubercle. Localizer was taken in axial, sagittal and coronal planes after making proper positioning of the patient, The MRI protocol consisted of sagittal T2W, T2WFFE, PDSPIR sequences; coronal PD, T2W, STIR sequences; Axial T2 Sequences, T2FSE. Additional 3D sequences and higher sequences were done whenever required. $140 \mathrm{~mm}$ field of view and a 212 x 168 matrix were taken. The slice thickness was $4 \mathrm{~mm}$ with $0.4 \mathrm{~mm}$ gap.

\section{Data Analysis}

Data was analyzed using SPSS (Statistical Package for Social Sciences) version 20 (IBM Inc. Chicago). Data was entered in excel sheet was imported to SPSS for statistical analysis. 
Variables were of qualitative nature and hence have been expressed as proportions in terms of percentages. Sensitivity, specificity and predictive values were calculated for MRI findings by comparing them with arthroscopic findings.

\section{RESULTS}

\section{Age and Sex}

There were 77 (77\%) male and 23 (23\%) female subjects out of the total 100 subjects. Right knee was injured in 55 (55\%) patients (44 males; 11 females) While left knee injury occurred in 45 (45\%) patients (33 males; 12 females). There were maximum numbers of patients in age group 21-30 years 39 (39\%) patients followed by $20(20 \%)$ patients in age group $11-20$ years and $18(18 \%)$ patients in age group of $31-40$ years. No patient younger than 10 years was observed. Sports related injuries (32\%) and roadside accident (30\%) accounted for the highest number of the injuries (62\%). It was followed by falls (23\%). Least number of cases with slipping injuries $(15 \%)$ was seen.

\section{MRI Analysis of Ligament Injury Patterns}

(complete and partial) in 100 patients is as follows: ACL tear: 50 tears; PCL Tears: 19; MCL tears: 17; LCL tears-16. Hence amongst the 102 ligamentous injuries in 100 subjects, ACL tears (complete/ partial) was the most commonly encountered pathology in this series (50\%) followed by PCL, MCL and LCL tears which did not show significant variation with each other. Site of ligament tear was most commonly encountered at femoral attachment (28 cases) followed by mid substance site (15 cases). Least common site was fibular/tibial attachment with 7 cases.

\section{Analysis of Meniscal Injury Patterns Identified on MRI}

Some patients had both medial and lateral meniscus tear. 49 cases were having abnormal signal intensity in medial meniscus tears. Incidence of grade III signal intensity ( medial meniscal tears) were seen in 30 cases, grade II signal intensity were seen in 10 cases followed by grade 1 signal intensity seen in 9 cases. There were 31 cases having abnormal signal intensity in lateral meniscus tears. Grade III signal intensity (tear) was seen in 17 cases followed by grade II signal intensity and grade 1 signal intensity 7 cases each.

\section{Type and Location of Grade III Meniscus Tears (LM + MM) on MRI}

Total No. of Grade III Medial meniscal tears observed on MRI were $30(30 \%)$ cases. Most common Type of tear was Bucket handle tear which comprised of 10 cases, followed by oblique meniscal tears and complex meniscal tears 7 cases each. Peripheral and radial tears constituted 4 cases and 2 cases respectively. Most common location of medial meniscal tear was in posterior horn, (19 cases), 11 of the cases showed tears in anterior horn which was second most common location. No tear was noted in the body of medial meniscus. Grade III Lateral meniscal tear seen in MRI were 17 cases and most common location was in posterior horn which was found in 11 cases followed by location in anterior horn seen in 6 cases. Most common type of tear was oblique tear seen in 7 cases followed bucket handle tear and complex tear seen in 4 cases each. Peripheral type of tear was seen 2 cases of lateral meniscus tears. No tear was noted in the body of lateral meniscus.

\section{Correlation of Clinical and MR Findings}

Correlation between Clinical and MR findings regarding presence or absence of ACL tear was - 80/100 (80\%), PCL tear: 93/100 (93\%), MCL tear: 84/100 (84\%), LCL tear: 90/100 (90\%), medial meniscal tear: - 70/100 (70\%), lateral meniscal tear: - $82 / 100(82 \%)$

\section{Arthroscopy}

Arthroscopic knee surgery was performed in 39 subjects out of the total 100 subjects. Rest of the patients were managed conservatively. Correlation of MRI findings and arthroscopy findings in 39 patients are listed below

Correlation of MRI Findings and Arthroscopic Findings $\mathrm{N}=39$.

\begin{tabular}{|ccccc|}
\hline MRI & ACL & PCL & MM & LM \\
Tear & 29 & 4 & 25 & 4 \\
Normal & 10 & 35 & 14 & 35 \\
Total & 39 & 39 & 39 & 39 \\
Findings on arthroscopy & ACL & PCL & MM & LM \\
Tear & 28 & 2 & 21 & 2 \\
Normal & 11 & 37 & 18 & 37 \\
Total & $\mathbf{3 9}$ & $\mathbf{3 9}$ & $\mathbf{3 9}$ & $\mathbf{3 9}$ \\
\hline \multicolumn{5}{c}{ Table 1. Findings on $\mathbf{M R I}$} \\
\hline
\end{tabular}

\begin{tabular}{|cc|}
\hline \multicolumn{1}{|c|}{ ACL Tear on MRI } \\
Anterior Cruciate Ligament (ACL) & No. of Subjects \\
Tear on MR & 29 \\
Tear on Arthroscopy (TP) & 28 \\
Normal on Arthroscopy (FP) & 1 \\
ACL Normal on MRI & No. of Subjects \\
Anterior Cruciate Ligament (ACL) & 10 \\
Normal on MR & 0 \\
Tear on Arthroscopy (FN) & 10 \\
Normal on Arthroscopy (TN) & \\
PCL Tear on MRI & No. of Subjects \\
Posterior Cruciate Ligament (PCL) & 4 \\
Tear on MR (TP) & 2 \\
Tear on Arthroscopy (TP) & 2 \\
Normal on Arthroscopy (FP) & PCL Normal on MRI \\
No. of Subjects \\
Posterior Cruciate Ligament (PCL) & 35 \\
Normal on MR & 0 \\
Tear on Arthroscopy (FN) & 35 \\
Normal on Arthroscopy (TN) & \\
\hline Table 2. Correlation of MRI and Arthroscopic Findings \\
in ACL \& PCL Tears \\
\hline
\end{tabular}

Agreement between MRI and arthroscopic findings regarding presence or absence of anterior cruciate ligament tears: sensitivity- 100\%, specificity-90.9\%, NPV- 100\%, PPV96.5\% and accuracy- 97.4\%. Correlation between MRI and arthroscopic findings regarding presence or absence of posterior cruciate ligament tears: sensitivity-100\% specificity-94.5\%, NPV- $100 \%$ PPV-50\% and accuracy94.8\%.

Agreement between MRI and arthroscopic findings regarding presence or absence of lateral meniscus tears: sensitivity- $100 \%$, specificity-94.5\%, NPV- 100\%, PPV-50\% and accuracy- (94.8\%) Agreement between MRI and arthroscopic findings regarding presence or absence of medial meniscal tears: sensitivity- $90.4 \%$, specificity- $66.7 \%$, 
NPV- 85.7\%, PPV-76\% and accuracy- 79.5\%. Agreement between MRI and arthroscopic findings regarding presence or absence of meniscal tears (combined MM and LM): sensitivity- 91.3\%, specificity-85.7\%, NPV- 96\%, PPV-72.4\% and accuracy- $87.3 \%$.

\begin{tabular}{|cc|}
\hline \multicolumn{2}{c|}{ Lateral Meniscus Tears on MRI } \\
Lateral Meniscus & No. of Subjects \\
Tear on MR & 4 \\
Tear on Arthroscopy (TP) & 2 \\
Normal on Arthroscopy (FP) & 2 \\
Lateral Meniscus Normal on MRI & No. of Subjects \\
Lateral Meniscus & 35 \\
Normal on MR & 0 \\
Tear on Arthroscopy (FN) & 35 \\
Normal on Arthroscopy (TN) & \\
Medial Meniscus Tears on MRI & No. of Subjects \\
Medial Meniscus & 25 \\
Tear on MR & 21 \\
Tear on Arthroscopy(TP) & 4 \\
Normal on Arthroscopy(FP) & \\
Medial Meniscus Normal on MRI & No. of Subjects \\
Medial Meniscus & 14 \\
Normal on MR & 0 \\
Tear on Arthroscopy(FN) & 14 \\
Normal on Arthroscopy(TN) & \\
\hline Table 3. Correlation of MRI and Arthroscopic Findings \\
In Meniscal Tears & \\
\hline
\end{tabular}

\section{DISCUSSION}

100 patients of knee injuries had undergone MR imaging. Arthroscopic knee surgery was performed in 39 out of the total 100 subjects. Rest of the 61 subjects underwent conservative management. Incidence of knee injuries was much more common in younger generation with peak incidence of $39 \%$ occurring in age group 21- 30 years in present study. Similar results have been shown by Clayton et $\mathrm{al}^{(7)} \mathrm{La}$ Prade et $\mathrm{al}^{(8)}$ and Incesu et al(9) with mean age varying from 24 - 36 years.

\section{Aetiology of Knee Injury}

There was almost equal incidence of knee injuries occurring due to sports (32\%) and roadside accidents (30\%) followed by falls $(23 \%)$ and slipping injuries $(15 \%)$. This is due to the fact present is a multi-specialty hospital with tertiary trauma care centre, so all kinds of patient referrals come to us thus almost equally distributing the subjects in above categories. In literature between $27 \%$ to $48 \%$ have been reported to be sports related (Kannus \& Jarvinen,(10) Nielsen \& Yde,(11) Ansari et al.(12) So incidence of sports related knee injuries of present study matches the incidence shown in these studies.

\section{Comparison of Correlation of Clinical and MRI Finding}

Correlation between Clinical and MR findings regarding presence or absence of ACL tear was - 80/100 (80\%), PCL tear: 93/100 (93\%), MCL tear: 84/100 (84\%), LCL tear: 90/100 (90\%), Medial meniscal tear: 70/100 (70\%), Lateral meniscal tear: 82/100 (82\%). Odqaard et al(13) observed correlation between clinical MR findings regarding presence or absence of ACL tears in $74 \%$ cases, PCL tear- $98 \%$ cases, MCL tear- $66 \%$ cases, LCL tear- $88 \%$ cases, Medial meniscus tear- $62 \%$ cases and LCL tear in $81 \%$ cases.
Analysis of Ligament and Meniscal Injury Pattern on MRI Examination

In present study out of 100 patients $50 \%$ showed ACL tears, 19\% showed PCL tears, (30\%) showed MM tears (GRADE III), $17 \%$ (grade III) showed LM-tears. Findings of present study matches with the study of Singh et al (14) and in variance with study of Ranjan and Mohammad.(15)

\begin{tabular}{|cccc|}
\hline $\begin{array}{c}\text { Injured } \\
\text { Ligament }\end{array}$ & $\begin{array}{c}\text { Present } \\
\text { Study N=100 } \\
\text { Percentage }\end{array}$ & $\begin{array}{c}\text { Singh et al } \\
\text { Study(14) } \mathbf{N = 1 7 3} \\
\text { Percentage }\end{array}$ & $\begin{array}{c}\text { Ranjan and } \\
\text { Mohamad(15) N=50 } \\
\text { Percentage }\end{array}$ \\
ACL & $50 \%$ & $45.09 \%$ & $76 \%$ \\
PCL & $19 \%$ & $5.78 \%$ & $6 \%$ \\
MM (Grade III) & $30 \%$ & $32.95 \%$ & $34 \%$ \\
LM (Grade III) & $17 \%$ & $16.18 \%$ & $22 \%$ \\
MCL & $17 \%$ & & $28 \%$ \\
LCL & $16 \%$ & $18 \%$ \\
\hline Table 4. Comparison of Ligament and Meniscal Tear \\
\multicolumn{4}{c}{ with Other Studies } \\
\hline
\end{tabular}

\section{Comparison of Grading of Meniscal Tears with Other Studies}

Some patients had both medial and lateral meniscus tear. 49 cases were having abnormal signal intensity in medial meniscus. Incidence of grade III signal intensity (medial meniscal tears) were seen in $30 \%$ cases, grade II signal intensity were seen in $10 \%$ cases followed by grade 1 signal intensity seen in $9 \%$ cases. There were $31 \%$ cases having abnormal signal intensity in lateral meniscus. Grade III signal intensity (tear) was seen in $17 \%$ cases followed by grade II signal intensity and grade 1 signal intensity 7\% cases each. Singh et al (14) observed Grade III signal intensity in $32.9 \%$ cases, Grade II signal intensity in $9.2 \%$ cases, and Grade I signal intensity in $11.3 \%$ cases in Medial Meniscus tears. Signal intensity in lateral meniscus tears observed was Grade III 16.18\%, Grade II 6.9\% and Grade I 8\%. Also, La Prada et al (8) reported that medial meniscal tears are twice as common as lateral meniscal tears which are in accordance with results of present study.

\section{Other Important Trauma Findings}

In present study 24 cases (24\%) cases bony contusions were seen out of 100 cases knee injuries. Most common site for bony contusion was femoral condyle and these findings are in agreement with Terzidis I P et al(16) study in which $27.8 \%$ of cases were seen with bony contusion and most common site for bony contusion was femoral condyle. Avulsion fracture was seen in 7 patients. One patient was seen with Segond fracture. There were $9 \quad(9 \%)$ patient with subluxation/translation of tibia and patella ( 6 cases of translation of tibia and 3 patients with subluxation of patella). One patient had osteochondritis dissecans and 3 (3\%) patients showed grade III iliotibial band injury Mansoor(17) et al reported $2.5 \%$ cases with grade 3 iliotibial band injury. Joint effusion was seen in $70(70 \%)$ cases on MRI in present study. Guler et al(18) did a study on 42 knees and observed that $81 \%$ cases showed joint effusion (mild to moderate level). 


\section{Arthroscopy}

In present study, 39 subjects underwent arthroscopic surgery.

\begin{tabular}{|cccccc|}
\hline ACL & $\begin{array}{c}\text { Present } \\
\text { Study }\end{array}$ & $\begin{array}{c}\text { Boeve B F } \\
\text { et al(19) }\end{array}$ & $\begin{array}{c}\text { Chen MC } \\
\text { et al }\end{array}$ (20) & $\begin{array}{c}\text { Winter } \\
\text { et al } \text { (21) }^{(21)}\end{array}$ & $\begin{array}{c}\text { Barronian } \\
\text { et al(22) }\end{array}$ \\
Sensitivity & $100 \%$ & $100 \%$ & $95 \%$ & - & - \\
Specificity & $90.9 \%$ & $96 \%$ & $89 \%$ & - & - \\
PPV & $96.5 \%$ & $80 \%$ & $88.5 \%$ & - & - \\
Accuracy & $97.4 \%$ & - & $92 \%$ & $90 \%$ & $82 \%$ \\
\hline \multicolumn{5}{|c}{ Table 5. Comparison of MRI and Arthroscopic Findings } \\
in ACL Injuries with Other Studies \\
\hline
\end{tabular}

These findings were in agreement with Boeve et study,(19) Chen $\mathrm{MC}$ et al(20) and in variance with Winter et al(21) and Barronian et al.(22)

\section{Comparison of MRI and Arthroscopic Findings in PCL Injuries with Other Studies \\ In case of PCL tears diagnostic accuracy for MR examination came out to be $94.8 \%$. Sensitivity and specificity for diagnosing PCL tear by MR was $100 \%$ and $94.5 \%$, Negative predictive value (NPV) was $100 \%$ making it a very good screening test. These findings match the results published by Laoruengthana et al(23) in 2012 which showed sensitivity $100 \%$, specificity of $97.1 \%$, accuracy $97.5 \%$ and negative predictive value $100 \%$.}

\section{Accuracy of MRI in Meniscal Injuries and Its Comparison with Other Studies}

Diagnostic accuracy of MRI was $79.5 \%$ for medial meniscus and $94.8 \%$ for lateral meniscus in present study which corresponds to studies done I by Glashow et al(24) (74\% for medial meniscus and 94\% for lateral meniscus), Rappeport et al(25) $\quad 77 \%$ for medial meniscus and $91 \%$ for lateral meniscus), Sharifah MI et al(26) (88 for medial menisci and $92 \%$ for lateral menisci) Sensitivity and Specificity for meniscal tears (both lateral and medial) were $91.3 \%$ and $85.7 \%$, respectively who present study. These values are similar to the study done by Chang et al.(27) Who reported the sensitivity and Specificity of $92 \%$ and $87 \%$ in diagnosis of meniscal tears respectively. Naranje $S$ et al(28) also shared same findings and observed sensitivity of $90 \%$ and specificity of $89 \%$.

\section{Predictive Value of MRI in Knee Injuries}

There was high negative predictive value (NPV) of MR examination (MM + LM) (96\%) in diagnosing meniscal tear as was in the case of ACL and PCL(100\% each) while the positive predictive value of MR examination was low in meniscal tears (MM + LM) (72.4\%). However positive predictive value of MR was $96.5 \%$ in diagnosing ACL tears. In the study by Barronian et al(22) the negative predictive value was $91 \%$ for menisci, whereas the positive predictive value was $65 \%$. Thus, it is evident again that MRI's negative predictive value makes it the investigation of choice in evaluation of knee injuries.

\section{False Positive and False Negative Cases}

There were 3 false positive cases of ligament tear ACL 1 cases and PCL 2 cases. This may be due to suboptimal selection imaging plan and partial volume averaging effect. Ranjan and Mohamad(15) also attributed low specificity of ACL tears due to above reason only. PPV of lateral meniscus tear was $50 \%$ and medial meniscus tear was 76\%. There were 6 false positive cases of meniscal tear on MR examinations in present study accounting for low positive predictive value of MR examination in meniscus tear diagnosed on arthroscopy. Out of these 6 false positive examinations, site of the 4 tears were located predominantly in the posterior horn and 2 was in the anterior horn. Posterior horn tears of menisci are likely to be missed on arthroscopy especially if anterior approach is used and if the menisci are not probed. Inferior surface of the meniscus is in particular vulnerable to this flaw in arthroscopy. Thus, acceptance of MRI findings as false positive is controversial. It is suggested that the reason of false positive and false negative meniscal lesion diagnosis was related to diagnostic errors in MRI as well as faults in arthroscopic evaluation. Levinsohn et al(29) from their study found that the accuracy of arthroscopy in identification of inferior surface tears of posterior horn of Medial Meniscus is as low as $45-65 \%$.

So present prospective study we found that Sensitivity and negative predictive value of MR examination for diagnosing ACL (sensitivity- 100\%, NPV- 100\%), PCL (sensitivity-100\%, NPV- 100\%) and meniscal tears (sensitivity- 91.3\%, NPV- 96\%) are quite high. So "one can rely on MRI to avoid diagnostic arthroscopy since MRI has a high sensitivity and high negative predictive value". The high sensitivity and negative predictive value of MR in this study makes it investigation of choice.
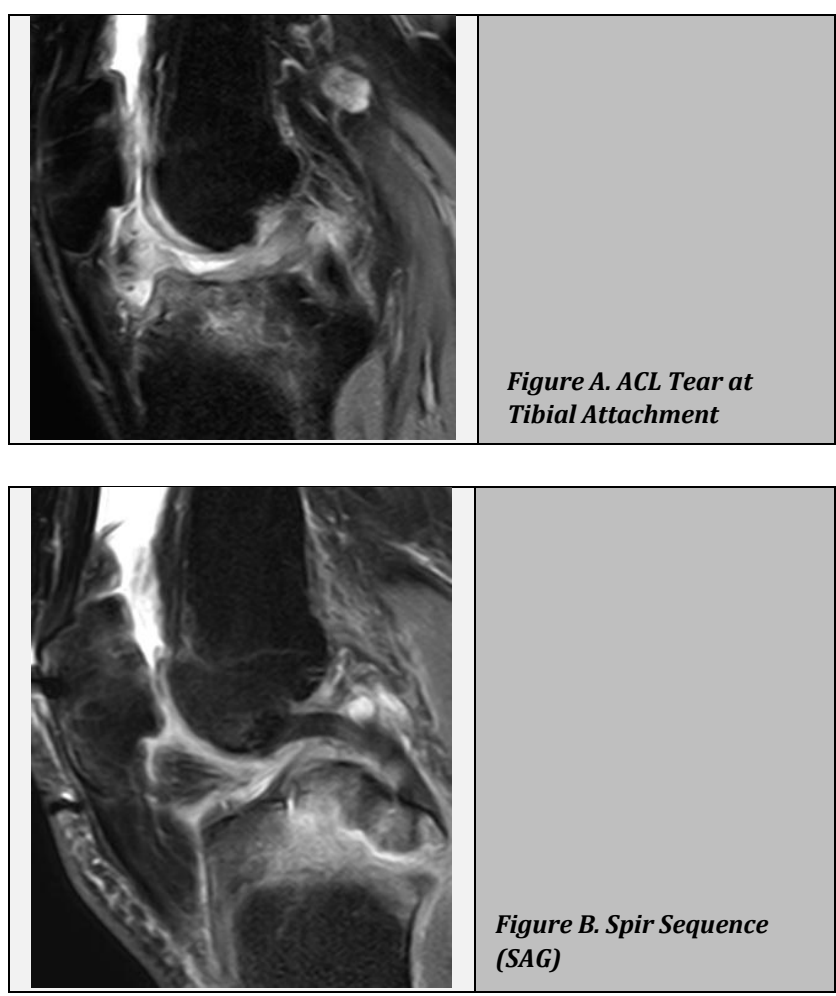


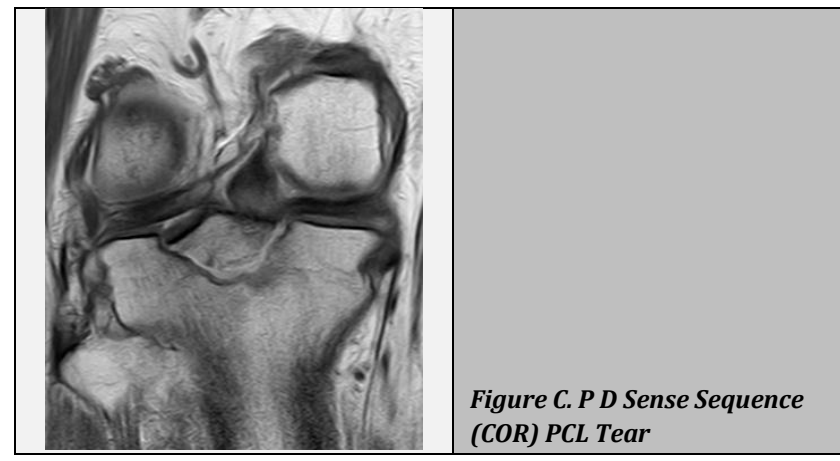

Hyperintense signal is seen in the fibres of ACL T2W sequence with avulsion fracture of tibia (segond fracture secondary sign) which can be seen on Dual sense sequence. There is associated joint effusion with bony contusion on tibial condyle seen in PD SPIR Sequence (sag). Hyperintense signal is seen in the fibres of PCL with loss of integrity of fibres seen on $\mathrm{T} 2 \mathrm{~W}$ ffe sequences. There is associated fracture of intercondylar region of tibia seen on P D spir sequences.
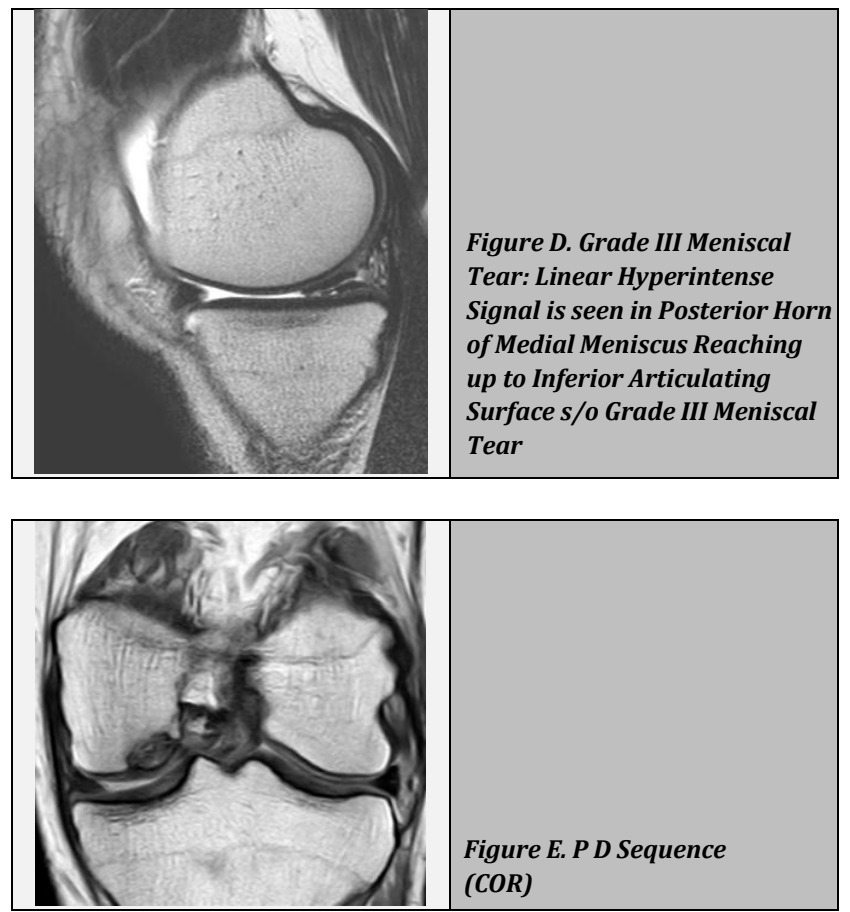

\section{Osteochondritis Dessicans}

Subchondral defect is seen along medial femoral condyle with fractured segment is lying along the medial femoral chondyle which can be seen in above figure.

\section{CONCLUSIONS}

100 cases of knee injury were evaluated with MRI examination. There was $77 \%$ males and $23 \%$ females. Massive number of patients were present in the age group of 21 to 30 years $39 \%$ followed by $20 \%$ in the age group of 11 20 year and $18 \%$ in age group of 31- 40 year. A good Correlation between MR findings and clinical findings was observed. MR diagnosis of 100 cases was as follows: in ACL tear 50\%; PCL tear 19\%; MCL tear 17\%; LCL tear 16\%; MM tear 49\%; LM tear 31\%; Sensitivity, specificity, NPV, PPV and accuracy of MRI in detecting the knee injuries taking arthroscopy as gold standard are as follows ACL- 100\%, 90.9\%, 100\%, 96.5\% and 97.4\%; lateral meniscus-100\%, $94.5 \%, 100 \%, 50 \%$ and $94.8 \%$; medial meniscus $90.4 \%$, $66.7 \%, 85.7 \%, 76 \%$ and $79.5 \%$ respectively. Correlation between MRI and arthroscopic findings regarding presence and absence of posterior cruciate ligament tear was highest with sensitivity $-100 \%$, specificity- $94.5 \%$, NPV- $100 \%$ and accuracy-94.8\%, There were 6 false positives for meniscus tear MR examinations in present study accounting for low positive predictive value (72\%) of MR examination. Posterior horn tears of menisci are likely to be missed on arthroscopy. Thus, acceptance of MRI findings as false positive is controversial. Out of 6 false positive cases of meniscus tear 4 were located in the posterior horn and 2 in the anterior horn.

MRI is a useful non-invasive modality having high diagnostic accuracy, sensitivity and negative predictive value making it a very reliable screening test for diagnosis internal derangements of knee joints. Hence MRI should be done in evaluation of patients with knee injuries saving them from unnecessary arthroscopies.

\author{
Abbreviation \\ MRI Magnetic Resonance Imaging. \\ ACL Anterior Cruciate Ligament. \\ MM Medial meniscus. \\ LM Lateral Meniscus. \\ LCL Lateral Collateral Ligament \\ PCL Posterior cruciate ligament. \\ MCL Medial collateral ligament. \\ T1W T1 weighted images. \\ T2W T2 weighted images. \\ PD Proton density. \\ FSE Fast Spin Echo. \\ FFE Fast Field echo. \\ SE Spin Echo. \\ SPIR Spectral Presaturation by Inversion Recovery. \\ STIR Short Tau Inversion Recovery. \\ NPV Negative Predictive Value. \\ PPV Positive Predictive Value.
}

\section{REFERENCES}

[1] MacMahon PJ, Palmer WE. A biomechanical approach to MRI of acute knee injuries. Am J Roentgenol 2011;197(3):568-77.

[2] Frobell RB, Lohmander LS, Roos HP. Acute rotational trauma to the knee: Poor agreement between clinical assessment and magnetic resonance imaging findings. Scand J Med Sci Sport 2007;17(2):109-14.

[3] Haapasalo H, Parkkari J, Kannus P, et al. Knee injuries in leisure-time physical activities: a prospective one-year follow-up of a finnish population cohort. Int J Sport Med 2007;28(1):72-7.

[4] El-Khoury GY, Manning TA, Tearse DS. MRI in the diagnosis of knee injuries. Iowa Orthop J 1993;13:70-8.

[5] Polly DW Jr, Callaghan JJ, Sikes RA, et al. The accuracy of selective magnetic resonance imaging compared with 
the findings of arthroscopy of the knee. J Bone Joint Surg Am 1988;70(2):192-8.

[6] Turner DA, Prodromos CC, Petasnick JP, et al. Acute injury of the ligaments of the knee: magnetic resonance evaluation. Radiology 1985;154(3):717-22.

[7] Clayton RAE, Court-Brown CM. The epidemiology of musculoskeletal tendinous and ligamentous injuries. Injury 2008;39(12):1338-44.

[8] LaPrade RF, Wentorf FA, Fritts $\mathrm{H}$, et al. A prospective magnetic resonance imaging study of the incidence of posterolateral and multiple ligament injuries in acute knee injuries presenting with a hemarthrosis. Arthrosc J 2007;23(12):1341-7.

[9] İncesu L, Dabak N, Belet $U$, et al, Comparison of magnetic resonance imaging (MRI) and arthroscopic findings in knee joint pathologies. Turkiye Klinikleri J Med Res 1997;15(1):21-5.

[10] Kannus P, Järvinen M. Incidence of knee injuries and the need for further care. A one-year prospective follow-up study. J Sports Med Phys Fitness 1989;29(4):321-5.

[11] Nielsen AB, Yde J. Epidemiology of acute knee injuries: a prospective hospital investigation. J Trauma 1991;31(12):1644-8.

[12] Ansari MZ, Ahee P, Iqbal MY, et al. Traumatic haemarthrosis of the knee. Eur J Emerg Med 2004;11(3):145-7.

[13] Odgaard F, Tuxoe J, Joergensen U, et al. Clinical decision making in the acutely injured knee based on repeat clinical examination and MRI. Scand J Med Sci Sports 2002;12(3):154-62.

[14] Singh JP, Garg L, Shrimali R, et al. MR Imaging of knee with arthroscopic correlation in twisting injuries. Indian J Radiol Imaging 2004;14(1):33-40.

[15] Rajan TS, Mohamed AJ. Evaluation of role of magnetic resonance imaging in knee joint injuries in correlation with arthroscopy. Int J Sci Study 2017. www.ijss-sn.com

[16] Terzidis IP, Christodoulou AG, Ploumis AL, et al. The appearance of kissing contusion in the acutely injured knee in the athletes. Br J Sports Med 2004;38(5):592-6.

[17] Mansour R, Yoong P, McKean D, et al. The iliotibial band in acute knee trauma: patterns of injury on MR imaging. Skeletal Radiol 2014;43(10):1369-75.

[18] Guler F, Kose 0, Erol B, et al. The prevalence of knee injuries ipsilateral to tibial shaft fractures and their impact on clinical outcome. Eur J Orthop Surg Traumatol 2015;25(1):141-8.

[19] Boeve BF, Davidson RA, Staab EV Jr. Magnetic resonance imaging in the evaluation of knee injuries. South Med J 1991;84(9):1123-7.

[20] Chen MC, Shih TT, Jiang CC, et al. MRI of meniscus and cruciate ligament tears correlated with arthroscopy. J Formos Med Assoc 1995;94(10):605-11.

[21] Winters K, Tregonning R. Reliability of magnetic resonance imaging of the traumatic knee as determined by arthroscopy. N Z Med J 2005;118(1209):U1301.

[22] Barronian AD, Zoltan JD, Bucon KA. Magnetic resonance imaging of the knee: correlation with arthroscopy. Arthrosc J Arthrosc Relat Surg 1989;5(3):187-91.

[23] Laoruengthana A, Jarusriwanna A. Sensitivity and specificity of magnetic resonance imaging for knee injury and clinical application for the Naresuan University Hospital. J Med Assoc Thai 2012;95(Suppl 10):S151-7.

[24] Glashow JL, Katz R, Schneider M, et al. Double-blind assessment of the value of magnetic resonance imaging in the diagnosis of anterior cruciate and meniscal lesions. J Bone Joint Surg Am 1989;71(1):113-9.

[25] Rappeport ED, Wieslander SB, Stephensen S, et al. MRI preferable to diagnostic arthroscopy in knee joint injuries. A double-blind comparison of 47 patients. Acta Orthop Scand 1997;68(3):277-81.

[26] Sharifah MIA, Lee CL, Suraya A, et al, Accuracy of MRI in the diagnosis of meniscal tears in patients with chronic ACL tears. Knee Surgery Sport Traumatol Arthrosc 2015;23(3):826-30.

[27] Chang CY, Hondar Wu HT, Huang TF, et al. Imaging evaluation of meniscal injury of the knee joint: a comparative MR imaging and arthroscopic study. Clin Imaging 2004;28(5):372-6.

[28] Naranje S, Mittal R, Nag $\mathrm{H}$, et al. Arthroscopic and magnetic resonance imaging evaluation of meniscus lesions in the chronic anterior cruciate ligamentdeficient knee. Arthrosc J 2008;24(9):1045-51.

[29] Levinsohn EM, Baker BE. Prearthrotomy diagnostic evaluation of the knee: review of 100 cases diagnosed by arthrography and arthroscopy. Am J Roentgenol 1980;134(1):107-11. 